\title{
Retrieval of Carbon Dioxide Vertical Concentration Profiles from Satellite Data using Artificial Neural Networks
}

A.R. CARVALHO ${ }^{1}$, F.M. RAMOS ${ }^{2}$, Laboratory for Computing and Applied Mathematics - LAC, Brazilian National Institute for Space Research (INPE), 12227-010 São José dos Campos, SP, Brazil.

J.C. CARVALHO ${ }^{3}$, National Water Agency - ANA, 70.610-200

Brasília, DF, Brazil.

\begin{abstract}
In this paper, we derive vertical distributions of carbon dioxide atmospheric concentration from satellite data using a retrieval algorithm based on an artificial neural network (ANN) technique. Sensitivity studies were made to select the most appropriate sensor channels. A MultiLayer Perceptron (MLP) ANN was implemented and tested for a large and diversified dataset. Here we focused on the retrieval of vertical Carbon Dioxide concentration profiles using SCIAMACHY channel 6 (1000-1700 nm) nadir measurements. The results show we can accurately and efficiently obtain carbon dioxide profiles by using this approach.
\end{abstract}

Keywords. Neural networks, Radiative Transfer Equation, $\mathrm{CO}_{2}$ vertical concentration profiles, Inverse problems.

\section{Introduction}

Carbon dioxide is the most important anthropogenic greenhouse gas. From 1832 to 2009 , the atmospheric $\mathrm{CO}_{2}$ concentration increased from 284 ppmv to $388 \mathrm{ppmv}$, or about 37\%, with most of the change occurring since 1970 [19]. It is becoming increasingly relevant to develop novel and better techniques to estimate concentration profiles of carbon dioxide. Until recently, information on sources and sinks on the global scale was derived from a precise but rather sparse network of about 100 ground stations (e.g., NOAA/ESRL). Retrievals from space based remote sensing instrumentation have the potential to overcome the limits of the surface network and constrain the inverse models. However, the retrieval of a long-lived and therefore well-mixed gas such as $\mathrm{CO}_{2}$ is challenging, because only small variations in the concentration distribution contain the necessary information on surface sources. Rayner and O'Brian estimate that we need a precision of $2.5 \mathrm{ppmv}$ ( $0.7 \%$ ) of $\mathrm{CO}_{2}$,

\footnotetext{
1 adenilson@lac.inpe.br

2 fernando@lac.inpe.br

3 joao.carvalho@ana.gov.br
}

Recebido em 30 Julho 2010; Aceito em 27 Outubro 2010. 
for monthly averaged column data at $8 \times 10^{\circ}$ spatial resolution, to get a performance comparable to the current network of ground stations [15]. Similar conclusions have been drawn by other studies [12].

Measuring $\mathrm{CO}_{2}$ from space is a new field of research and only a few relevant studies exist. $\mathrm{CO}_{2}$ retrieval feasibility and sensitivity studies have already been performed for the Scanning Imaging Absorption Spectrometer for Atmospheric Chartography (SCIAMACHY) ([4] and [5]). The first $\mathrm{CO}_{2}$ measurements results from SCIAMACHY also have been presented in [5]. The SCaning Imaging Absorption spectroMeter for Atmospheric ChartographY (SCIAMACHY) sensor is part of the atmospheric chemistry equipment aboard the ESA's Envisat environmental satellite, launched in March 2002. The SCIAMACHY instrument is a multichannel spectrometer that measures the scattered, reflected and transmitted solar radiation. It covers the spectral wave band of $240-2385 \mathrm{~nm}$ at nadir, limb and both solar and lunar occultation observational modes, with a spectral resolution varying from $0.2-1.6 \mathrm{~nm} \mathrm{[2].} \mathrm{The} \mathrm{sensor} \mathrm{spatial} \mathrm{resolution} \mathrm{depends} \mathrm{on} \mathrm{the} \mathrm{spectral} \mathrm{range}$ and on the orbital position. For channel 6, the spatial resolution is $30 \times 60 \mathrm{~km}^{2}$, corresponding to an integration time of $0.25 \mathrm{~s}$. As a result, this sensor has a good potential to estimate carbon dioxide and other greenhouse effect gases, using the principle of reflected radiation in the NIR. Preliminary results showed that we can use channel 6 (1000-1750 nm) for inference of the $\mathrm{CO}_{2}$ and $\mathrm{CH}_{4}$ column in the atmosphere [3].

Atmospheric and surface physical properties determine the radiance measured by an instrument at the top of the atmosphere. We describe this dependence by means of the Radiative Transfer Equation (RTE). The mathematical model for the RTE used in this study takes into account the effects of anisotropic scattering but ignores the thermal emission term because it is irrelevant in the near-IR spectral region of 1000-1750 $\mathrm{nm}$ (channel 6). The expression for the RTE used, in this case, has the same form as given in [17] and [13] and can be expressed as follows

$$
\mu \frac{d I(z, \mu, \varphi)}{d z}=-c(z) I(z, \mu, \varphi)+\frac{b(z)}{4 \pi} \int_{0}^{2 \pi} \int_{-1}^{1} p\left(z, \mu, \mu^{\prime}, \varphi, \phi^{\prime}\right) I\left(z, \mu^{\prime}, \varphi^{\prime}\right) d \mu^{\prime} d \varphi^{\prime}
$$

with

$$
\begin{aligned}
a(z) & =a_{M}(z)+\sum_{i=1}^{k} \alpha_{i} \eta_{i}(z) \\
b(z) & =a_{M}(z)+b_{R}(z) \\
c(z) & =a(z)+b(z)
\end{aligned}
$$

where $\mu$ is the cosine of the zenith angle $\theta$. The boundary conditions at the top and the bottom of the atmosphere lead to

$$
\begin{aligned}
I^{-}\left(z_{0}, \mu, \varphi\right) & =\delta\left(\mu-\mu_{0}, \varphi-\varphi_{0}\right) \pi F \\
I^{+}(0, \mu, \varphi) & =\frac{A}{\pi} \int_{0}^{2 \pi} \int_{0}^{1} I^{-}\left(0, \mu^{\prime}, \varphi^{\prime}\right) \mu^{\prime} d \mu^{\prime} d \varphi^{\prime}
\end{aligned}
$$


where $I^{-}\left(z_{0}, \mu, \varphi\right)$ is the downwelling radiance at $z_{0}$ and $I^{+}(0, \mu, \varphi)$ is the upwelling radiance at the ground. For sake of simplicity the dependence on the wavelength $\lambda$ is omitted and a Lambertian surface albedo is assumed. The other symbols used in the equation along their meanings are shown in Table 1 below:

Table 1: Symbols used in the RTE

\begin{tabular}{|c|c|}
\hline Symbol & Meaning \\
\hline$I$ & total radiance (i.e., radiation field) \\
\hline$z$ & altitude; \\
\hline$z_{0}$ & altitude at the top of atmosphere \\
\hline $\begin{array}{l}\mu, \mu^{\prime} \\
\mu_{0}\end{array}$ & $\begin{array}{l}\text { cosines of zenith angles } \theta, \theta^{\prime} \\
\text { cosine of the solar zenith angle } \theta_{0} ;\end{array}$ \\
\hline$\varphi, \varphi^{\prime}$ & $\begin{array}{l}\text { azimuthal angles in relation to the line-of-sight } \\
\text { projection on the earth surface }\end{array}$ \\
\hline$\varphi_{0}$ & $\begin{array}{l}\text { azimuthal angle of the Sun in relation to the line-of-sight } \\
\text { projection on the earth surface }\end{array}$ \\
\hline$\kappa$ & number of the trace gases \\
\hline$c$ & total extinction coefficient \\
\hline$a$ & $\begin{array}{l}\text { total absorption coefficient (sum of trace gas and particle } \\
\text { absorption coefficients) }\end{array}$ \\
\hline$b_{R}$ & Rayleigh-scattering coefficient \\
\hline$b_{M}^{n}$ & particle-scattering coefficient \\
\hline & total scattering coefficient (sum of Rayleigh and particle-scattering) \\
\hline $\begin{array}{l}p_{M} \\
p\end{array}$ & $\begin{array}{l}\text { Rayleigh-scattering phase function } \\
\text { total scattering phase function }=\left(b_{R} p_{R}+b_{M} p_{M}\right) /\left(b_{R}+b_{M}\right)\end{array}$ \\
\hline & trace gas absorption cross sections for all relevant gases \\
\hline$n_{i}^{i}$ & concentration of trace gases \\
\hline & particle absorption coefficient \\
\hline$\pi F \mu_{0}$ & solar flux at the top of the atmosphere \\
\hline & spectral reflectance (Lambertian) of the Earth's surface \\
\hline
\end{tabular}

Here, we use the RTE to estimate the trace gas concentration profiles from suitable gas absorption bands within the NIR transmission window. In this case, the vertical temperature profile is determined by an independent inversion procedure and we need only to retrieve the gas mixing ratio profile that, in the formulation presented in [17], is part of the equation. The knowledge of the atmospheric thermodynamic structure and of the concentration of the atmospheric components allows, through the RTE, to calculate the radiance and brightness temperature measured by the sensors on the satellite. The associated inverse problem is to estimate the state of atmosphere from measured radiances. In the present case, we want by solving the inverse problem, to estimate the $\mathrm{CO}_{2}$ atmospheric concentrations, from radiances measured by the SCIAMACHY's sensor channels. This inverse problem presents many difficulties. First, the RTE to be inverted is a Fredholm integral equation of the first kind, whose ill-posed nature is well known see [21]. Second, within the RTE, the concentration intervenes through the transmission function, which is highly nonlinear. Finally, the $\mathrm{CO}_{2}$ signal intensities are weak, even in the more sensitive channels, and have the order of magnitude of the instrument noise [8].

Thus, most classical estimation techniques are inappropriate. The goal of this paper is to present an inversion algorithm, based on Multilayer perceptron (MLP) artificial neural networks (ANNs), which retrieve $\mathrm{CO}_{2}$ vertical concentration profiles from satellite measurements. The use of neural networks within this context is a natural approach that brings a satisfactory answer to these difficulties. For example, being nonlinear by construction, neural networks avoid the need of linearization, a frequent source of inaccuracies in classical inversion methods. More- 
over, since the learning phase, which is the more intense computational task, runs off-line and only once, the computational efficiency of the inversion process is high. Also, the proposed approach easily handles problems such as the strong ill-posed nature of the nonlinear radiative inverse problem, the presence of noise and the high-dimensionality of sensor's data set. Neural networks have been successfully employed to estimate: thermodynamical variables (see, for example, [9], moisture profiles and surface characteristics [18], CO mixing ratios [7], ozone mixing ratios [20] and in the direct modeling of radiative parameters [6].

This paper is organized as follows. The physical problem, the forward model and a brief review of ANN applications to the atmospheric constituents inversion is shown in the section 1 . In the section 2 we explain the technique used for channel selection and give more information about the inverse model and its training, validation and testing dataset. We present the results and pertinent discussions in section 3. Finally, conclusions and perspectives are given in section 4 .

\section{Materials and Methods}

As a first step, we examined the performance of the radiative transfer model (the forward model) that was used in the inversion. Based on these studies we decided to exploit SCIAMACHY's near-infrared channel 6. As a second step, we performed sensitivity studies to separate the most suitable subchannels within this spectral range. Finally, we developed an ANN based model to retrieve the trace gas concentration profiles from (synthetic and real) remote sensing data. To this end, we employed synthetic radiances simulated by SCIATRAN [16] for the ANN learning process. SCIATRAN is a radiative transfer forward model developed for retrieval of atmospheric trace gas concentrations, aerosol and cloud parameters, and surface reflectance from the spectral radiance measurements of the SCIAMACHY/ENVISAT1 and GOME/ERS-2 UV-Vis-NIR multichannel spectrometers. SCIATRAN solves the RTE using a finite differences method for a plane-parallel, pseudo-spherical or spherical atmosphere, vertically inhomogeneous, considering multiple scattering. The wavelength range covered by the radiative transfer model is $175-2380 \mathrm{~nm}$ (see [16] for more details).

In our study, the SCIATRAN version 2.1. was adjusted in such a way that all radiative transfer calculations were performed in a spherical atmosphere (i.e., we consider all effects due to the sphericity of the Earth's atmosphere), based on full multiple scattering radiative tranfer simulations, in the nadir mode, with a $0.045^{\circ}$ instrument field of view size, azimuth angle of $76.35^{\circ}$ and a global albedo of 0.3 . The retrieval of atmospheric $\mathrm{CO}_{2}$ abundance is made from SCIAMACHY observations under clear sky and clean air conditions. The pixels produced by the sensor showing clouds are identified and discarded prior to our study.

\subsection{Channel selection}

We can use an ANN approach, in principle, to map any input vector space to any output vector space. However, in practice, the data representation significantly affects the quality of the results. We may use dimension reduction techniques to 
present not only a more compact representation but also more relevant information to the input of the ANN (see, for example, [11]). The "curse of dimensionality" stipulates that it is hard to apply a statistical technique to high-dimension space data. The number of parameters (the weights in the ANN) increases with the number of inputs. This can allow excessive degrees of freedom in the neural interpolator and introduce uninformative data (i.e., noise or spectral information unrelated to retrieved quantities), which may distort the learning process [1].

Thus, the goal of dimension reduction is to present to the ANN the most relevant information from initial raw data (i.e., noisy physical measurements). We can reduce the dimensionality of the input data through feature extraction (a transformation, linear or not, of raw data) or using feature selection through selection of specific channels in the input data [14]. Here we chose the feature selection approach. For the retrieval of one geophysical variable, we select channels that are, as far as possible, uniquely sensitive to this one atmospheric parameter. By studying the derivatives of the radiances with respect to each geophysical parameter (RTE Jacobians), it is possible to inspect the common information between measured radiances and geophysical variables. Nevertheless, we need to make a trade-off between reducing data dimensionality and preserving the information content in the data to compensate the effects of noise. We based our feature selection method on the study of finite difference estimates of the partial derivatives of the measured radiance with respect to the gas concentration (integrated along the column or at a given pressure level), for each wavelength. This approach is called "sensitivity analysis". Particularly, we focused on the infrared channels presenting certain desirable features such as a low noise level in the signal; high sensitivity to changes in the concentration profile; minimum contamination by other gas absorption lines such as $\mathrm{H}_{2} \mathrm{O}, \mathrm{O}_{3}, \mathrm{~N}_{2} \mathrm{O}, \mathrm{CO}$; and low sensitivity to the temperature profile.

So, the results presented in this manuscript have been derived using $\mathrm{CO}_{2}$ absorption features in channel $6(1000-1750 \mathrm{~nm})$ with 507 spectral lines, which are not affected by an ice-layer, as their detectors operate at higher temperatures. Figure 1 shows the spectral sensitivity of channel 6 for $\mathrm{CO}_{2}, \mathrm{~N}_{2} \mathrm{O}$ and $\mathrm{CH}_{4}$ when we add $10 \%$ to the respective reference concentration profiles as suggested in [10].

We note that highly sensitive $\mathrm{CO}_{2}$ lines in channel 6 are mainly located within two spectral windows, around $1200 \mathrm{~nm}$ and from 1580 to $1650 \mathrm{~nm}$. We also took into account the cross section effect of the $\mathrm{N}_{2} \mathrm{O}$ and $\mathrm{CH}_{4}$ lines for the $\mathrm{CO}_{2}$ retrieval. The final result, with the selected spectral lines is depicted in Table 2. Overall, 21 lines have been chosen for $\mathrm{CO}_{2}$ retrieval.

Table 2: SCIAMACHY channels used to recover the $\mathrm{CO}_{2}$ vertical concentration profile $(\mathrm{nm})$.

\begin{tabular}{|lllllll|}
\hline 1219.00 & 1291.60 & 1438.10 & 1441.10 & 1532.80 & 1568.30 & 1569.78 \\
1571.26 & 1572.74 & 1574.22 & 1577.18 & 1580.14 & 1581.62 & 1584.60 \\
1597.90 & 1599.38 & 1600.86 & 1602.34 & 1603.82 & 1606.80 & 1608.28 \\
\hline
\end{tabular}



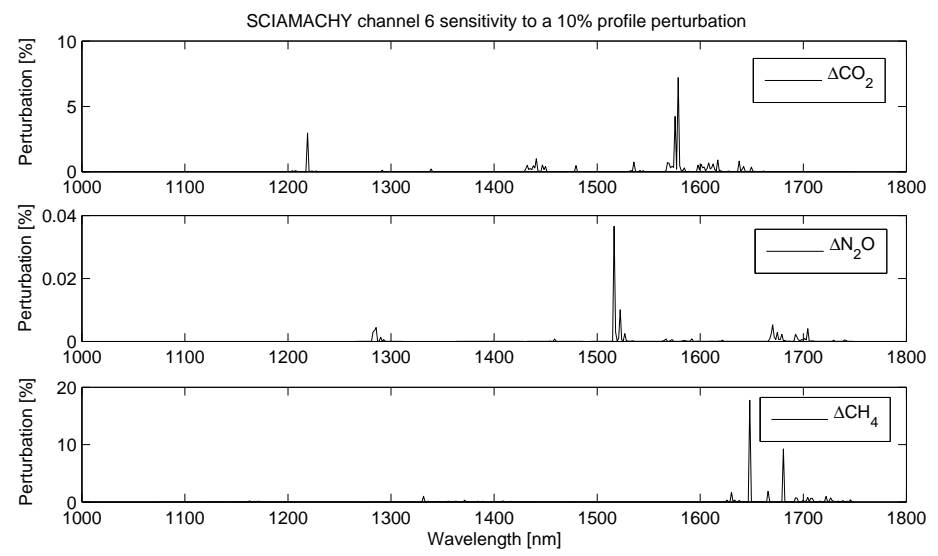

Figure 1: Sensitivity in percent to a $10 \%$ profile perturbation vs. wavelength in the 1000-1750 nm SCIAMACHY window (channel 6).

\subsection{ANN training and testing}

The next step, after selecting the most promising channels, is the generation of suitable training patterns to serve as input for our ANN based inverse model. The procedure to create the training database is as follows: For a given gas, we select from SCIATRAN's climatological database, a reference concentration profile $P_{0}=\left[C_{01}, C_{02}, \ldots, C_{0 m}\right]$, where $\mathrm{m}$ is the number of vertical points. We then run the forward model for $P_{0}$ producing a radiance vector $R_{0}=\left[R_{01}, R_{02}, \ldots, R_{0 n}\right]$, where $n$ is the number of selected channels or wavelengths chosen previously. We modify $P_{0}$ to generate a profiles set $P_{\text {base }}$ comprising new profiles $P_{j}$, with $j=$ $-J_{\max }, \ldots, J_{\max } \sim 50$ through the relation $P_{j}=P_{0} \times\left(1+\frac{j}{100}\right)$

We repeatedly run the forward model to obtain the corresponding $R_{j}$ vectors. Then we derive three more profile sets from $P_{b a s e}$ by perturbing stochastically the profiles $P_{j} \in P_{\text {base }}$ to generate three new state concentration vectors through $P_{\sigma}^{k}=$ $P_{j} \times(1+\sigma \times \mu)$, where $\sigma$ is the noise standard deviation and $\mu$ is a random variable taken from a Gaussian distribution with zero mean and unitary variance. The values for $\sigma$ we used were $0,0.01,0.05$ and 0.1 . We proceed this way to get a dataset as representative as possible of the real state of the atmosphere. In this study, we considered different reference profiles $P_{0}$ representing the climate in mid-latitudes, sub-Arctic, and equatorial regions of the globe for the two well-defined seasons of winter and summer.

Finally, each dataset $\left(R_{a}\right.$ and $\left.R_{b}\right)$ was divided into three subsets following as suggested by Haykin [11]:(I) Training set, with 2232 samples ( 92\%), comprising the training patterns to be used during the ANN training phase;(II) Validation set, with 120 samples $(\sim 5 \%)$, to be used in the cross validation to evaluate the ANN performance; (III) Generalization set, with 72 samples $(\sim 3 \%)$, employed for the effective test of the ANN, known as generalization test. In our experiments, weights and biases of all ANN models have been randomly initialized with values 
drawn from a $[-1,1]$ uniform distribution. A momentum term was specified to filter out high frequency disturbances on the error surface. The initial values for learning rate and momentum term as well as the activation function used in our experiments are 0.125 and 0.40 for the logarithmic activation function and 0.015 and 0.8 for the Gaussian. We considered data sets with $10 \%, 5 \%, 1 \%$ and $0 \%$ (noiseless) of zero mean additive white Gaussian noise (AWGN) added to the synthetic data to analyze the performance of the ANNs in the retrieval of vertical carbon dioxide profiles. The mean generalization errors of the simulation results for each trace gas were calculated through:

$$
\text { Error }=\sqrt{\sum_{i=p_{s}}^{p_{t}}\left(C_{i}^{\text {exact }}-C_{i}^{\text {model }}\right) / N}
$$

where $N$ is the number of profile sample points, $p_{s}$ and $p_{t}$ are, respectively, the pressure level at the surface and the top of the atmosphere (TOA). All the atmospheres used during the learning and the testing phases are described by 50 atmospheric concentration measurement points (LOWTRAN levels up to $0.000036 \mathrm{hPa}$ or 120 $\mathrm{km}$ height) and the corresponding selected radiances computed by SCIATRAN. To solve the problem of the $\mathrm{CO}_{2}$ concentration retrieval we developed an algorithm, written in Java language, that implements a Multilayer perceptron ANN ([11] and [1]). All experiments were conducted under the Linux operating system, in a microcomputer processor with AMD Athlon (tm) 64 Processor $3200+, 1.53 \mathrm{GHz}$ and $1 \mathrm{~Gb} \mathrm{MB}$ of RAM.

\section{Results and Discussion}

Table 3 presents the precise combinations of parameters used in each simulation, as well as a summary of statistics for $\mathrm{CO}_{2}$ dataset. Different number of hidden neurons has been considered for each ANN architecture. We perform 10 experimental runs of the model for each topological configuration found. Next, we grouped the generated profiles and produced a resulting average profile. The standard deviation at the end of each round of experiments is also displayed in the table below.

Table 3: Results for the retrieval of $\mathrm{CO}_{2}$ vertical concentration profile with $1 \%$ Additive White Gaussian Noise (AWGN)

\begin{tabular}{|c|c|c|c|}
\hline Number of layers & $\begin{array}{c}\text { Neurons in } \\
\text { hidden layers }\end{array}$ & $\begin{array}{c}\text { Generalization } \\
\text { Error (in ppmv) }\end{array}$ & $\begin{array}{c}\text { Standard deviation } \\
\text { (in ppmv) }\end{array}$ \\
\hline 2 & $13-5$ & 1.6309 & 0.5944 \\
\hline 2 & $15-10$ & 1.6523 & 0.7180 \\
\hline 1 & 30 & 1.6684 & 0.8223 \\
\hline
\end{tabular}

Figure 2 shows the results of the generalization tests for the model in comparison with synthetic radiance data (here called the true profile model). The results obtained with the MLP network for $\mathrm{CO}_{2}$ noisy data are in excellent agreement with the true model. The reconstructed profiles are homogeneous and present error of 
the order of $\pm 1 \%$ ( $\pm 3.8 \mathrm{ppmv}$ in $380 \mathrm{ppmv})$, for the lower troposphere. We can consider this result as the error due to the neural network model since there is no noise in training data. When we corrupt the data by adding $1 \%$ gaussian noise the profile could be reconstructed with a global error less than $2 \%$ and presented a smooth and homogeneous shape.

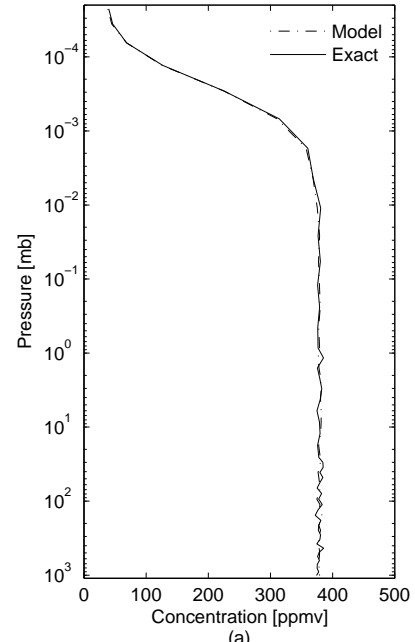

(a)

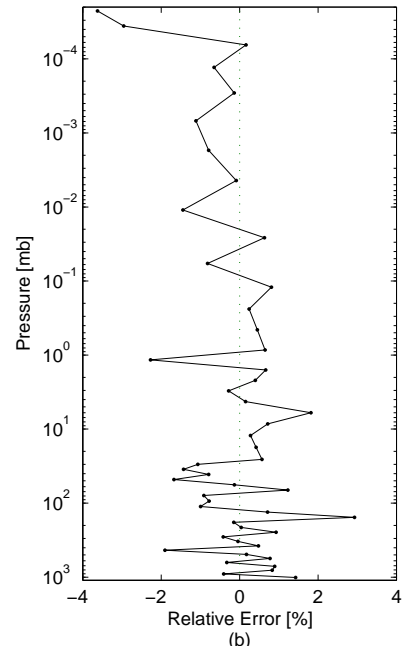

(b)

Figure 2: (a) $\mathrm{CO}_{2}$ mixing ratio (in ppmv) retrieval results in tropical region for data corrupted with $1 \%$ white gaussian noise using MLP ANN with 2 hidden layers having 13 and 5 neurons in each layer, respectively. (b) Retrieved profile error (in percent).

In particular, the overall performance of the model for $\mathrm{CO}_{2}$ retrieval in the boundary layer region is also satisfactory presenting a rms error of $\pm 1 \%( \pm 3.8$ ppmv in $380 \mathrm{ppmv}$ ) for noisy data. If we look in the upper troposphere and in the most part of the stratosphere the error remains lower than about $2 \%$ if we handle data with or whithout noise. A good match with the experimental profile curve is also obtained in the upper layers (mesosphere and part of the thermosphere) but increases substantially in the higher altitudes (above $100 \mathrm{~km}$ ) where the errors reach a maximum of $4 \%$. We validate the retrieved results with available in situ data from Flask measurements obtained at Mauna Loa Observatory, Hawaii at latitude $19.54^{\circ}$ $\mathrm{N}$, longitude $155.58^{\circ} \mathrm{W}$ and elevation $3397.0 \mathrm{~m}$ [19]. The data is distributed evenly throughout the year 2005 and amounted to a set of 12 observations. In the figure 3 a comparison is made between data obtained by the proposed inversion model and the in-situ data collected at the observatory. In general the errors obtained by the model are close to the threshold established by [15] for the maximum error allowed to estimate $\mathrm{CO}_{2}$ integrated column (see section 1).

The precision (random error) of the retrieved $\mathrm{CO}_{2}$ Mixing Ratio due to instrument noise is $\sim 1 \%$ for the spectral fitting window used for this study (for an albedo 

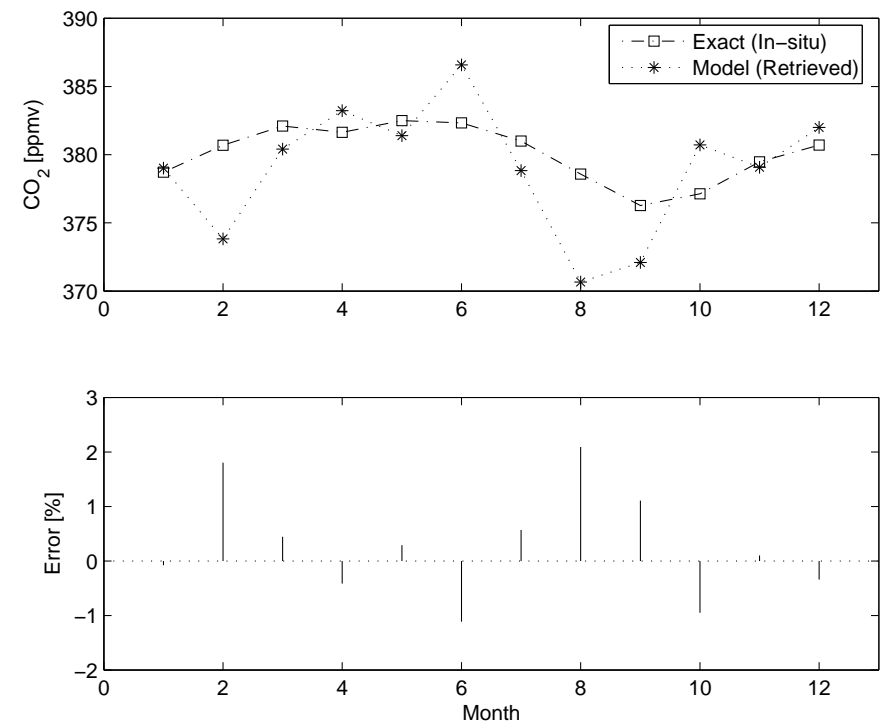

Figure 3: (Top) $\mathrm{CO}_{2}$ mixing ratio (in ppmv) retrieval results for Mauna Loa, Hawaii using MLP ANN with 2 hidden layers having 13 and 5 neurons in each layer, respectively. (Bottom) Residual Fit error (in percent).

of 0.3 and a solar zenith angle of $53^{\circ}$ ) [3]. Comparisons between the $\mathrm{CO}_{2}$ predicted by the ann based model and those observed by SCIAMACHY along the year 2005 in the selected region show, in general, good agreement. The fit residuum is detected to within $3 \%$ with the mean difference between the $\mathrm{CO}_{2}$ distributions being 1-3\% and the standard deviation approximately $0.75 \%$. The correlation between the time series of the SCIAMACHY and the monthly model averages is typically $\sim 0.61$ what indicates a moderated ability of the inversion algorithm to retrieve seasonal changes in $\mathrm{CO}_{2}$ concentrations. However, SCIAMACHY detects a seasonal cycle amplitude about 2 times larger than predicted by the model, which can not be explained yet.

From this study the overall precision and bias of the retrieved Mixing Ratios are estimated to be close to $1.0 \%$ and $3.0 \%$ respectively. It also must be re-stressed that scaling factors were not applied at any stage to the retrieved $\mathrm{CO}_{2}$ Mixing Ratios as they have been in other studies. Whilst these results are encouraging they are still not of the desired quality for inverse modelling. It is hoped that further improvements to the retrieval algorithm, through better calibration of the SCIAMACHY data and by improving the quality of the input data set used by the ann model in the training, will overcome this issue in the future. 


\section{Conclusions}

Atmospheric $\mathrm{CO}_{2}$ vertical concentration profiles are retrieved in the tropics at latitude and longitude coordinates (19.54N,155.58W) using SCIAMACHY's channels 6 $(1000-1700 \mathrm{~nm})$ near-infrared nadir measurements. The inversion method relies on a nonlinear regression inference scheme using neural networks. For this, we applied a multilayer perceptron (MLP) architecture with supervised learning and the backpropagation algorithm for the network training. Sensitivity studies were performed and a methodology to select the most appropriate subchannels for the retrieval was created.

The use of neural networks was able to solve this difficult inverse problem even when the data were contaminated with noise. The overall precision and bias of the retrieved vertical profiles are estimated to be close to $1.0 \%$ and lower than $3.0 \%$ respectively. A rough estimate of the mean relative error is about $1.5 \%$ for the synthetic data and $2 \%$ for the experiment with satellite data. Finally, the satellite retrievals have been compared with ground-based in-situ measurements from NOAA ESRL flasks network.

From this work, two advantages of the use of neural networks in the retrieval of trace gases became clear. First, after the training phase, the reconstruction algorithm is much faster (in the order of milliseconds) than the classical inversion methods. Second, it can be easily implemented in a parallel environment. A future work could be to compare the performance of the model with other operational approaches and use this results to produce $\mathrm{CO}_{2}$ source and sinks distribution maps. More studies, an improved calibration, and algorithm refinements are needed to enable accurate retrievals of $\mathrm{CO}_{2}$ columns.

Resumo. Neste paper, derivamos distribuições verticais de concentração atmosférica de dióxido de carbono a partir de dados de satélite utilizando um algoritmo de inversão baseado em uma técnica de redes neurais artificiais (RNA). Estudos de sensibilidade foram efetuados para selecionar os canais mais apropriados do sensor. Uma RNA Perceptron de múltiplas camadas foi implementada e testada para um amplo e diversificado conjunto de dados. Aqui, focamos a recuperação de perfis verticais de concentração de dióxido de carbono usando medidas em modo nadir no canal 6 do sensor SCIAMACHY (1000 - $1700 \mathrm{~nm})$. Os resultados mostram que podemos de obter perfis de $\mathrm{CO}_{2}$ de maneira eficiente e precisa por meio desta abordagem.

\section{References}

[1] C.M. Bishop, "Neural Networks for Pattern Recognition", Oxford University Press, 1995.

[2] H. Bovensmann, J.P. Burrows, M. Buchwitz, J. Frerick, S. Noel, V.V. Rozanov, SCIAMACHY - Mission Objectives and Measurement Modes, J. Atmos. Sci., 56 (1999), 127-150.

[3] M. Buchwitz, J.P. Burrows, Retrieval of $\mathrm{CH}_{4}, \mathrm{CO}$, and $\mathrm{CO}_{2}$ total column amounts from SCIAMACHY near-infrared nadir spectra: Retrieval algorithm 
and first results, in "Proceedings of SPIE 5235, Remote Sensing of Clouds and the Atmosphere VIII", K. P. Schafer and A. Comeron and M. R. Carleer and R. H. Picard (Editors), 375-388, 2004.

[4] M. Buchwitz, S. Noel, K. Bramstedt, V.V. Rozanov, M. Eisinger, H. Bovensmann, S. Tsvetkova, J.P. Burrows, Retrieval of trace gas vertical columns from SCIAMACHY /ENVISAT near-infrared nadir spectra: first preliminary results, Advances in Space Research, 34 (2004), 809-814.

[5] M. Buchwitz, et al., Atmospheric methane and carbon dioxide from SCIAMACHY satellite data: initial comparison with chemistry and transport models, Atmos. Chem. Phys., 5 (2005), 941-962.

[6] F. Chevallier, F. Cheruy, N. A. Scott, A. Chedin, A neural network approach for a fast and accurate computation of longwave radiative budget, J. of Applied Meteorology, 37 (1998), 1385-1397.

[7] C. Clerbaux, J. Hadji-Lazaro, S. Payan, C. Camy-Peyret and G. Megie, Retrieval of CO columns from IMG/ADEOS spectra, IEEE Transactions on Geoscience and Remote Sensing, 37 (1999), 1657-1661.

[8] C. Crevoisier, A. Chedin and N.A. Scott, AIRS channel selection for $\mathrm{CO}_{2}$ and other trace-gas retrievals, Q. J .Roy. Meteor. Soc., 129 (2004), 2719-2740.

[9] J. Escobar-Munoz, A. Chedin, F. Cheruy, N.A. Scott, Réseaux de neurones multi-couches pour la restitution de variables thermodynamiques atmosphériques á l'aide de sondeurs verticaux satellitaires, Comptes Rendus de l'Académie des Sciences de Paris, 317 (1993), 911-918 (in French).

[10] L. Garand, D.S. Turner, Radiance and jacobian intercomparison of radiative transfer models applied to HIRS and AMSU channels, Journal of Geophysical Research, 106, No. D20, (2001), 24017-24031.

[11] S. Haykin, "Neural Networks. A Comprehensive Foundation", Macmillan, New York, NY, 1994.

[12] S. Houweling, F.M. Breon, I. Aben, C. Rodenbeck, M. Gloor, M. Heimann, P. Ciais, Inverse modeling of $\mathrm{CO}_{2}$ sources and sinks using satellite data: A synthetic inter-comparison of measurement techniques and their performance as a function of space and time, Atmos. Chem. Phys., 4 (2004), 523-538.

[13] J. Lenoble, "Radiative Transfer in Scattering and Absorbing Atmospheres: Standard computational procedures", A. Deepak Publishing, Hampton, Virginia, 1985 .

[14] F. Rabier, N. Fourrie, D. Chafai, P. Prunet, Channel selection methods for infrared atmospheric sounding interferometer radiances, Quart J. Roy. Meteor. Soc., 111 (2002), 974-975. 
[15] P.J. Rayner, R.M. Law., D.M. O’Brien, T.M. Butler, A.C. Dilley, Global observations of the carbon budget. 3. Initial assessment of the impact of satellite orbit, scan geometry, and cloud on measuring $\mathrm{CO}_{2}$ from space, Advances in Computer Science, Journal of Geophysical Research, 107 (2002), doi: 10.1029/2001JD000618.

[16] V.V. Rozanov, M. Buchwitz, K.U. Eichmann, R. de Beek, J.P. Burrows, SCIATRAN - a new radiative transfer model for geophysical applications in the 240-2400 nm spectral region: The pseudo-spherical version, New York: John Wiley \& Sons, Advances in Space Research, 29, No. 11 (2002), 1831-1835.

[17] V.V. Rozanov, D. Diebel, R.J.D. Spurr, J. P. Burrows, GOMETRAN:A radiative transfer model for the satellite project GOME - the plane-parallel version, J. Geophys. Res., No. 102 (1997), 16683-16695, doi:10.1029/96JD01535.

[18] E.H. Shiguemori, J.D.S. da Silva, H.F. de Campos Velho, J.C. Carvalho, Neural Network based Models in the Inversion of Temperature Vertical Profiles from Satellite Data, Inverse Problems in Engineering, England, 14, No. 5 (2006), $543-556$.

[19] P. Tans, et al., "Trends in Atmospheric Carbon Dioxide - Mauna Loa", NOAA/ESRL, www.esrl.noaa.gov/gmd/ccgg/trends, 2009.

[20] S. Turquety, J. Hadji-Lazaro, First satellite ozone distributions retrieved from nadir high-resolution infrared spectra, Geophysical Research Letters., 29 (2002).

[21] S. Twomey, "Introduction to the Mathematics of Inversion in Remote Sensing and Indirect Measurements", Dover Publications Inc., 243 pp., 1977. 